\title{
Norois
}

Environnement, aménagement, société

$228 \mid 2013$

La patrimonialisation des paysages de l'eau dans

l'ouest de la France

\section{Aménagements portuaires et batellerie traditionnelle, composantes de ressources territoriales en Val de Loire}

Harbours Facilities and Inland Waterway Boating, Components of Territorial Resources in the Loire Valley (France)

Benoît Pin, Jean-Baptiste Rigot et Sylvie Servain

\section{(2) OpenEdition}

Journals

Édition électronique

URL : http://journals.openedition.org/norois/4771

DOI : 10.4000/norois. 4771

ISBN : 978-2-7535-2918-2

ISSN : $1760-8546$

Éditeur

Presses universitaires de Rennes

Édition imprimée

Date de publication : 30 septembre 2013

Pagination : 53-63

ISBN : 978-2-7535-2883-3

ISSN : 0029-182X

Référence électronique

Benoît Pin, Jean-Baptiste Rigot et Sylvie Servain, « Aménagements portuaires et batellerie

traditionnelle, composantes de ressources territoriales en Val de Loire », Norois [En ligne], 228 | 2013, mis en ligne le 30 septembre 2015, consulté le 01 mai 2019. URL : http://journals.openedition.org/ norois/4771 ; DOI : 10.4000/norois.4771 


\title{
Aménagements portuaires et batellerie traditionnelle, composantes de ressources territoriales en Val de Loire
}

\author{
Harbours Facilities and Inland Waterway Boating, \\ Components of Territorial Resources in the Loire Valley (France)
}

\author{
Benoît Pin ${ }^{* a}$, Jean-Baptiste Rigot ${ }^{\mathrm{b}}$, Sylvie SERvain ${ }^{c}$
}

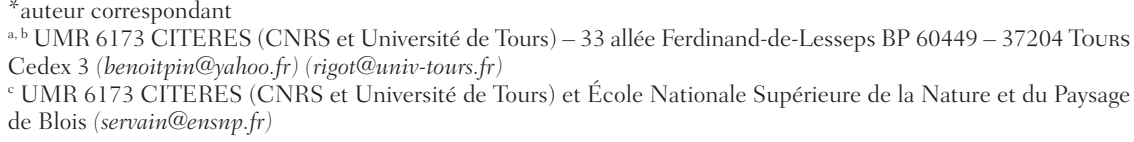

Résumé : À la fin du siècle dernier, le Val de Loire a fait l'objet d'un processus de patrimonialisation acté par l'inscription au patrimoine mondial de l'UNESCO. Parmi les motifs paysagers valorisés par cette patrimonialisation, les ports et bateaux traditionnels sont mobilisés en tant que ressources pour l'animation touristique et culturelle.

Ces objets qui font référence à un passé récent ont été restaurés ou reconstruits de manières inégales, en agrégeant leurs fonctions initiales à de nouvelles ou plus simplement en recherchant la satisfaction de nouvelles pratiques. Les formes de ces objets sont donc variées mais leur intégration aux projets de développement locaux présente des tendances générales communes. Ainsi, peu importe la fidélité à l'ancien, les aménagements pour la navigation et les bateaux intègrent de manière constante les projets de développements locaux assis sur des ressources territoriales.

Leur dispersion sur l'ensemble du linéaire ligérien et leurs potentialités touristiques et culturelles en font aujourd'hui des objets patrimoniaux incontournables du Val de Loire comme en témoignent des actions portées à l'échelle des départements. Ainsi, ces objets sont-ils des composantes d'un modèle de développement original en milieu rural.

\begin{abstract}
At the end of the past century, the heritage of the Loire Valley was recognised and listed as a UNESCO World Heritage Site. Some of the valued landscape assets, harbours and traditional boats are used as a resource for festive and cultural activities.

These objects referring to the recent past have been unequally restored or rebuilt: new functions were created or added to the original ones. The shapes of these objects are vary but their integration into local development projects present general common trends. Navigation facilities and boats constantly integrate local development projects based on territorial resource with no fidelity to the past.

Their distribution over the whole Loire Valley and their tourist and cultural potentials have turned them into unmissable objects as actions are carried out across the several departments of the Loire Valley. Thus, these objects seem to be constant components of an original model for the development in rural areas.
\end{abstract}

Mots clés : navigation - patrimoine - tourisme - fleuve - Loire

Keywords: navigation - heritage - tourism - river - Loire 


\section{INTRODUCTION}

Le patrimoine de la vallée de la Loire, surtout identifié pour ses composantes architecturales et «monumentales », a connu depuis la fin des années 1980, une réelle extension sémantique agrégeant des patrimoines vernaculaires anonymes aux grands sites. Cet enrichissement, acté en novembre 2010, par l'inscription du Val de Loire au patrimoine mondial de l'Unesco, au titre des paysages culturels vivants et évolutifs, est à replacer dans l'émergence du tourisme rural du début des années 1990. Le corollaire de cette filiation est la reconnaissance de «petits » patrimoines à l'échelle de territoires plus ou moins définis. Ici, nait la notion de «ressource territoriale » (Gumuchian et Pecqueur, 2007).

Cette évolution récente du patrimoine du Val de Loire dans une perspective de mutation du profil touristique des lieux traduit la plasticité de la notion en fonction des besoins ${ }^{1}$. Ceci se matérialise de deux manières. La première correspond à une évolution de ce qui peut être recouvert par le vocable de patrimoine. Ainsi, au fur et à mesure du processus de patrimonialisation, les objets valorisés peuvent être différents, de nouveaux objets s'agréger à ceux initialement reconnus pour constituer une offre touristique originale (Pecqueur, 2006). La seconde se matérialise dans les variations de forme des objets patrimoniaux reconstruits, entre recherche d'une certaine fidélité à l'objet initial d'un côté et créations inspirées des objets anciens de l'autre.

Si la grande majorité des auteurs s'intéressant à la question du patrimoine introduisent leur propos sous l'angle de sa construction et de son invention (Choay, 1992, Nora, 1997, Greffe, 1999), il s'agit ici de questionner les processus faisant suite aux premières étapes de la patrimonialisation décrites par Di Méo (2008). Ces derniers illustrent une démarche de développement touristique qui, sans aller jusqu'à une transformation du territoire en décor, peut aller jusqu’à la « re-création touristique d'un passé idéalisé » (Brunel, 2007). Ce mouvement participe de la singularisation et la différenciation des territoires dans une logique de développement. Le projet est alors construit autour d'actions valorisant cet objet consensuel qu'est le patrimoine

1. « Ce qui est caractérisé comme "extension patrimoniale" ou multiplication des problèmes d'environnement, c'est d'ailleurs le plus souvent cette capacité de reconfiguration liée aux évolutions des usages du patrimoine et de l'environnement » (Melé, 2006). en tant que ressource territoriale (Landel et Senil, 2009). Ceci aboutit à valoriser un ensemble que l'on peut qualifier d'actifs patrimoniaux spécifiques au territoire, constituant le produit vendu, association interdépendante de produits et services (Pecqueur, 2006).

Dans la vallée de la Loire, les recherches menées sur le patrimoine (Davodeau 2008, Verdelli 2008, Bonnefond et al., 2009) comme les études des gestionnaires ${ }^{2}$ ont montré sa diversité et les mutations d'usage liées au processus de patrimonialisation (Chiappero, 1996). Parmi les héritages fluviaux, les aménagements portuaires et les bateaux sont particulièrement intéressants à considérer puisque les usages économiques qui y sont associés, d'utilitaires, sont devenus, du moins en partie, touristiques et de loisirs et que des actions de construction ou de rénovation ont été mises en œuvre (Pin, 2010). Ceci nous a amené, dans le cadre d'un projet de recherche s'inscrivant dans la continuité de travaux sur les paysages ligériens, à nous interroger sur l'intérêt porté à la dimension patrimoniale des objets liés à la navigation et constituant une offre touristique originale. Premièrement, il s'agissait de mettre en perspective les reconstructions d'aménagements portuaires (ports, cales, quais) et des bateaux sous l'angle du respect de l'authenticité ou de la simple utilisation de l'image. Deuxièmement, nous souhaitions interroger l'action publique visant ces objets. L'identification des objectifs poursuivis vise à évaluer l'émergence et la consolidation d'un modèle de développement local alternatif, mobilisant une ressource territoriale construite.

Le terrain d'investigation choisi est un tronçon de Loire moyenne, en aval de Tours (Indre-et-Loire), et deux communes rurales, Chouzé-sur-Loire et Bréhémont (figure 1), où des projets et des activités mobilisant le patrimoine lié à la navigation ont été initiés.

La méthode adoptée couple des données contemporaines, issues de l'analyse d'entretiens d'acteurs, de questionnaires auprès d'habitants, de dossiers techniques et de documents de communication ainsi que de données historiques antérieures au $\mathrm{xx}^{\mathrm{e}}$ siècle.

2. La revue 303 a ainsi publié un numéro spécial sur la Loire en 2003 et en 2012 un numéro intitulé " Val de Loire, patrimoine mondial », qui regroupent des contributions de chercheurs mais également de professionnels (conservateur du patrimoine, architecte) et d'élus, contribuant à dresser un portrait du patrimoine du Val de Loire. 
Figure 1 : Carte du terrain d'étude

Location map

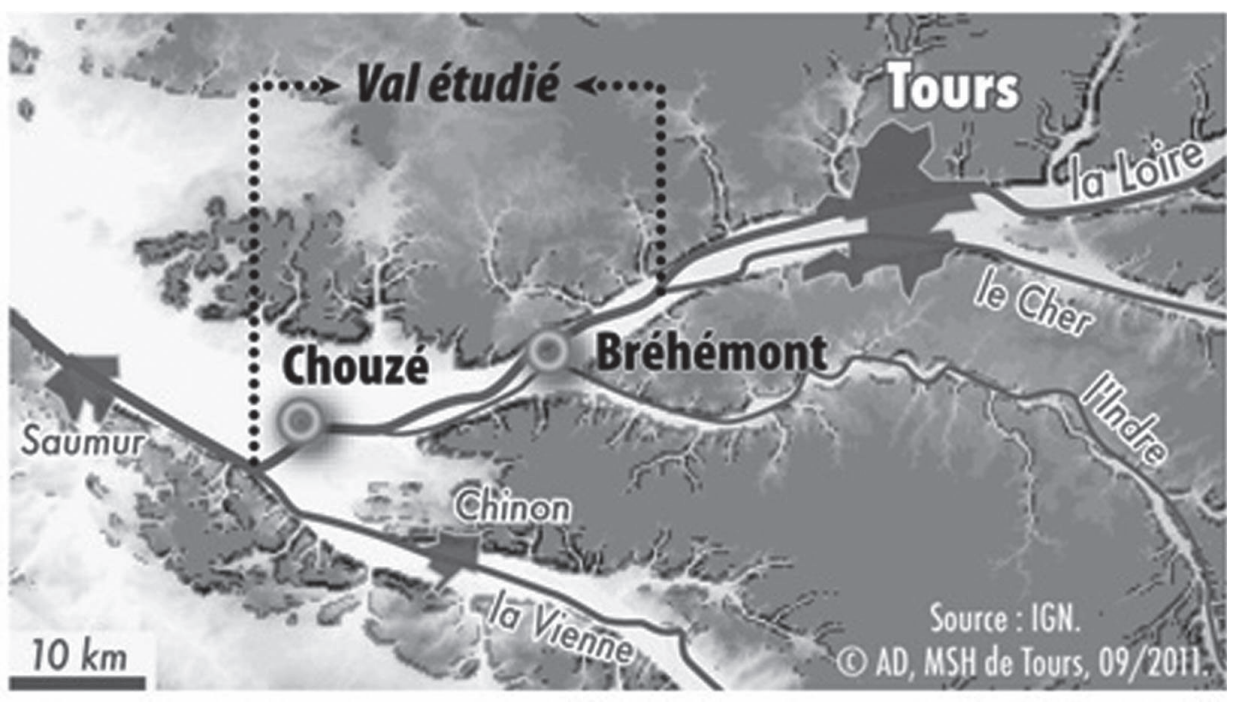

Une vingtaine d'entretiens semi-directifs ont été menés auprès d'un panel d'acteurs de l'animation culturelle et touristique (associations de batellerie, constructeurs de bateaux, archéologues fluviaux, présidents de communauté de communes, prestataires du développement touristique), afin de les interroger sur l'intérêt patrimonial des objets concernés par notre recherche; ceci en abordant les projets dans lesquels ils sont impliqués ou ceux dont ils ont connaissance. Le traitement de ces entretiens a consisté en une transcription puis une analyse qualitative des discours, des représentations et des pratiques (Kaufmann, 2001). Le questionnaire, à destination des habitants, est quant à lui composé d'une trentaine de questions fermées ou ouvertes s'intéressant à l'appropriation, aux pratiques et aux représentations du paysage en tant que patrimoine. Ce sont les composantes visuelles mentionnées par les habitants comme des éléments de ce paysage typique et patrimonial qui ont été plus spécifiquement utilisées dans cet article : il s'agissait de faire ressortir l'intérêt patrimonial porté aux objets. L'exploitation des 70 questionnaires, collectés dans les communes de Bréhémont et de Chouzé-sur-Loire, a été conduite en deux temps; tout d'abord la construction d'une typologie des réponses aux questions ouvertes puis la réalisation de tests statistiques multivariés (AFC et $\mathrm{ACM}$ ) croisant les questions thématiques et les questions sur le contexte.

La dernière source de données contemporaine est constituée par les dossiers techniques et documents de communication des prestataires de l'animation culturelle et touristique. Leur analyse est fondée sur la recherche systématique de termes et de discours afin de pouvoir caractériser les projets liés au patrimoine de la navigation et d'identifier les éléments mobilisés. Enfin, des données d'archives, iconographiques et textuelles, portant sur la Loire et la navigation (du $\mathrm{XVIII}^{\mathrm{e}}$ et au début du $\mathrm{Xx}^{\mathrm{e}}$ siècle), ont été collectées et analysées; ceci afin de placer dans un contexte historique les projets et démarches réalisés autour du patrimoine.

Après avoir présenté le contexte historique de la navigation en Loire et les aménagements induits, nous détaillerons plusieurs expériences de reconstruction du patrimoine et nous interrogerons sur sa conservation et sa valorisation dans les projets de développement.

\section{Navigation en Loire TOURANGELLE, DE L'HISTOIRE À LA RESTAURATION D'UN PATRIMOINE FLUVIAL}

L'inscription du Val de Loire par l'UNESCO au titre des paysages culturels vivants et évolutifs marque la reconnaissance d'un patrimoine agglomérant différents objets. La navigation, qui est peu mentionnée dans le dossier d'inscription, devient, a posteriori, un des éléments majeurs de la valorisation du patrimoine ligérien (Pin, 2010), s'appuyant sur un discours lui donnant une importance historique réelle.

Pourtant la référence à un passé où la navigation constituait une composante principale de la vallée 
de la Loire est à nuancer. Ainsi, selon l'expression de Bernard Chevalier (1986), historien des villes, notamment ligériennes, la Loire a toujours été un « mauvais chemin ». D’après cet auteur, ce fleuve n'est intéressant pour les échanges commerciaux qu'en raison de la présence d'une route qui la longe. Par ailleurs, l'axe navigable qu'elle détermine ne semble pas avoir été, contrairement aux idées préconçues, déterminant pour l'apparition des centres urbains et l'activité économique qui les accompagne, en tout cas avant le XIv siècle. L'image d'un fleuve navigué et navigable de tout temps qui est utilisée aujourd'hui dans le processus de valorisation patrimoniale, doit être questionnée au regard de l'histoire.

La navigation en Loire est avérée depuis longtemps comme l'attestent des travaux archéologiques (Fouillet et al. 2010), mais aucun élément matériel ne montre que ce transport est suffisamment important pour constituer un vecteur du développement économique avant le $\mathrm{XI}^{\mathrm{e}}$, et surtout le XIV ${ }^{\mathrm{e}}$ siècle. À partir du $\mathrm{XI}^{\mathrm{e}}$, la Loire est animée d'abord par les échanges suscités par les grandes abbayes bénédictines qui se relèvent de l'épisode normand. Mais ces échanges sont limités et ce sont surtout les terroirs rattachés aux abbayes qui vont susciter le développement du val (Chevalier, 1986). En revanche, à partir du XIV siècle l'activité fluviale semble prendre une véritable importance économique, comme en témoigne la fondation de la "Communauté des marchands fréquentant la rivière de Loire et autres fleuves descendant en icelle » (Jeanson et Mantellier, 1987). Cette corporation est un groupement d'intérêt dont les fonctions sont de nature parapublique : entretien du chenal navigable, balisage annuel, élimination des péages seigneuriaux. Ce développement de la navigation s'accompagne d'aménagements portuaires, en particulier en milieu urbain. Ainsi à Tours, on compte une vingtaine de ports à la fin du Moyen Âge dont trois semblent véritablement résulter d'un aménagement concerté de la part de la communauté urbaine (Durremberger 1999). En milieu rural, ces ouvrages, qui sont de simples rampes d'échouage et de débarquement, sont également présents (Duremberger, 1999, Le Nevez et al., 2007).

Le trafic sur le fleuve à cette époque se limite à quatre postes principaux (bois, blé, vin, sel). Il s'accrôit et se diversifie fortement à partir de la fin du
$\mathrm{XV}^{\mathrm{e}}$ jusqu'au milieu du XVII ${ }^{\mathrm{e}}$ siècle. La baisse du coût du fret y est pour beaucoup, ainsi que le développement industriel des villes et l'accroissement de la production agricole en Beauce et du vignoble en Loire. À partir du milieu du XVII ${ }^{\mathrm{e}}$ siècle, le trafic décline; la communauté des marchands perd la responsabilité de l'entretien du chenal navigable en 1682 au profit de l'administration royale puis la corporation disparaît en 1787. Par la suite, l'arrivée du chemin de fer et la sécurisation de la route donnent un coup d'arrêt définitif à l'activité fluviale. Ainsi le trafic passe, entre Tours et Orléans, de 400000 tonnes en 1840-1845 à 150000 t en 1853 , puis de 25000 t en 1880 à 1100 en 1893 .

Ce rapide panorama historique permet de nuancer l'importance de la navigation en Loire et notamment son rôle dans le développement économique du Val. Cette question nous intéresse car c'est une image inverse qui est aujourd'hui véhiculée, reposant à juste titre sur l'histoire de la fin du Moyen Âge et de la Renaissance. Cet arrangement trouve-t-il un écho dans les expériences de restauration du patrimoine associé à la navigation? Est-ce une simple matérialisation d'une instrumentalisation du patrimoine dans une perspective de valorisation? Les positions sont variées, qu'il s'agisse des aménagements portuaires ou de la batellerie traditionnelle.

Concernant les aménagements portuaires, certains documents d'archives témoignent de leur évolution et surtout du soin apporté à leur conservation au cours des projets de restauration. Ainsi ces documents montrent que les ports prennent leur aspect actuel entre 1856 et 1866. À Bréhémont en particulier, le port a été totalement reconstruit jusqu'en 1869 (figure 2). Notons à cet égard, que le profil de l'ancien quai (figure 3), constitué d'un haut mur presque vertical au pied duquel s'étendait une pente douce pavée, n'avait rien de commun avec les talus de perrés qui seront systématiquement élevés tout au long du XIX ${ }^{\mathrm{e}}$ siècle (Verdier et Guilbaud, 2003).

L'aspect des ports n'a que peu changé depuis cette époque, et les projets de restauration dont ils font l'objet ne vont pas infléchir la tendance. Ces projets financés par la collectivité reposent sur des dynamiques locales spécifiques s'appuyant notamment sur la présence d'associations inscrites dans le mouvement dit du « renouveau de la marine de Loire ». Ce vocable désigne de manière courante le développement important d'associations dont l'objet est la 


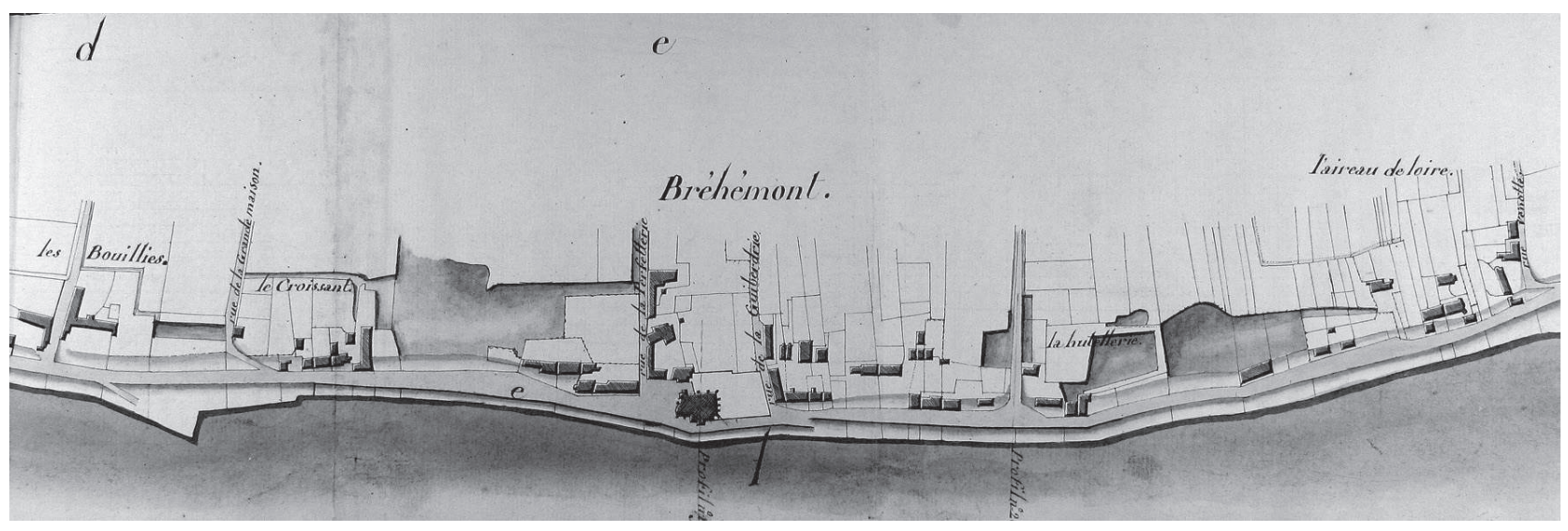

Figure 2 : Le port de Bréhémont, extrait des croquis de la levée nord de la Loire, 1840 ADIL II/3.1.26 The Brehemont harbour, extracted sketches of the north dyke, 1840 ADIL II/3.1.26

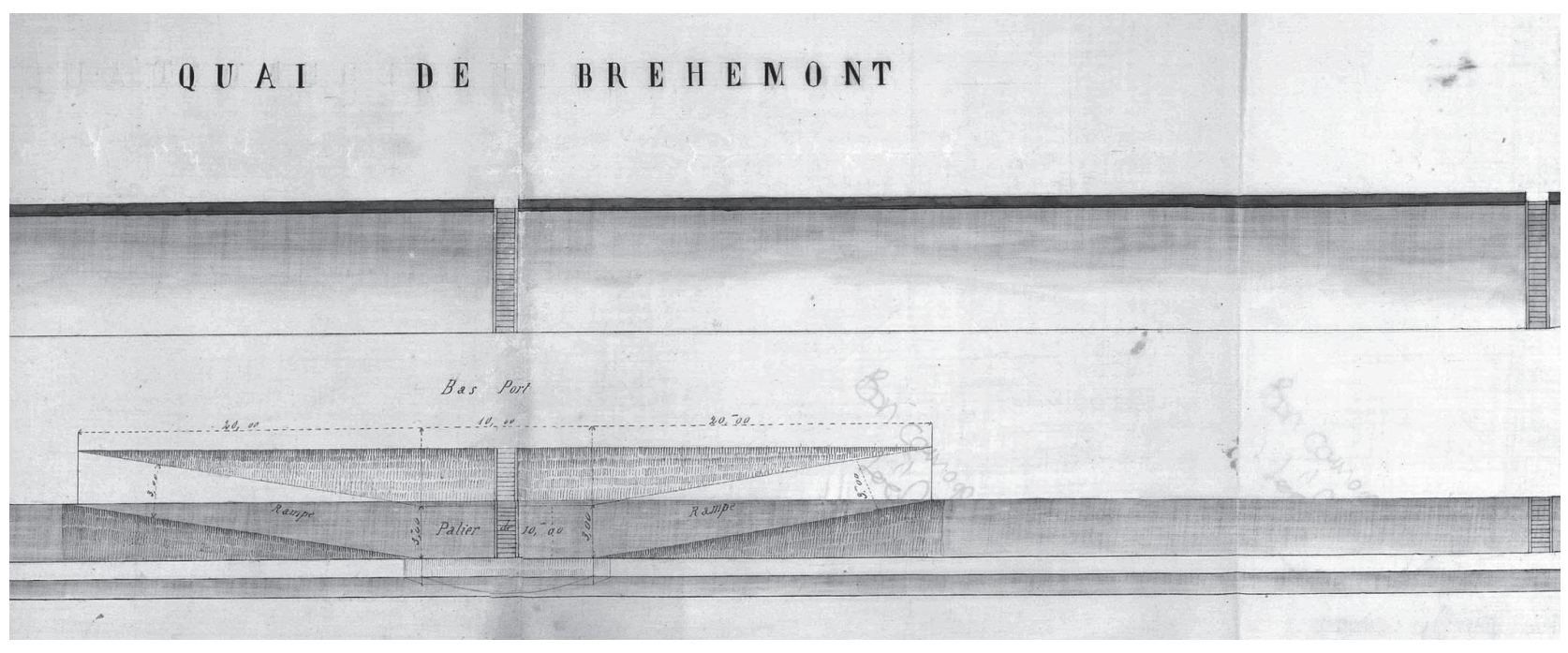

Figure 3 : Relevé du profil de l'ancien quai de Bréhémont effectué par l'ingénieur responsable du chantier avant sa reconstruction en 1869 (Source : Archives Départementales d'Indre-et-Loire, côte S4940)

Cross-section of the Bréhémont old embankment drawn by the engineer responsible for the site before its reconstruction in 1869 (source: Indre-et-Loire departmental archives, S4940)

navigation à bord de bateaux « traditionnels » dont elles assurent la (re)construction. Ces associations sont aussi à l'origine du développement de fêtes marinières. C'est ainsi que le port de Bréhémont a été restauré grâce à l'appui d'un financement départemental. Ce dernier a été choisi pour plusieurs raisons par le conseil général d'Indre-et-Loire dont la présence d'associations, une dynamique locale touristique mais également la localisation dans un bassin de navigation. Ce dernier étant intéressant d'un point de vue paysager et patrimonial tout autant que touristique. L'importance de ce contexte dans le choix d'intervention du conseil général éclaire la restauration de ce port où un soin particulier a été porté à la fidélité à l'ancien et à la satisfaction des usages actuels. Si le profil du port n’a pas été transformé comme en témoignent la comparaison entre une carte postale du début du siècle dernier et une photographie de la situation actuelle (figure 4), on comprend néanmoins l'importance d'un contexte favorable à la valorisation du patrimoine dans les procédures de restauration.

À Chouzé-sur-Loire, la situation est différente puisque le port a fait l'objet de travaux, à l'initiative de la commune, antérieurs à ceux réalisés dans le cadre de la restauration du patrimoine fluvial par le conseil général d'Indre et Loire. Le projet de rénovation comprend un aménagement paysager et urbain 


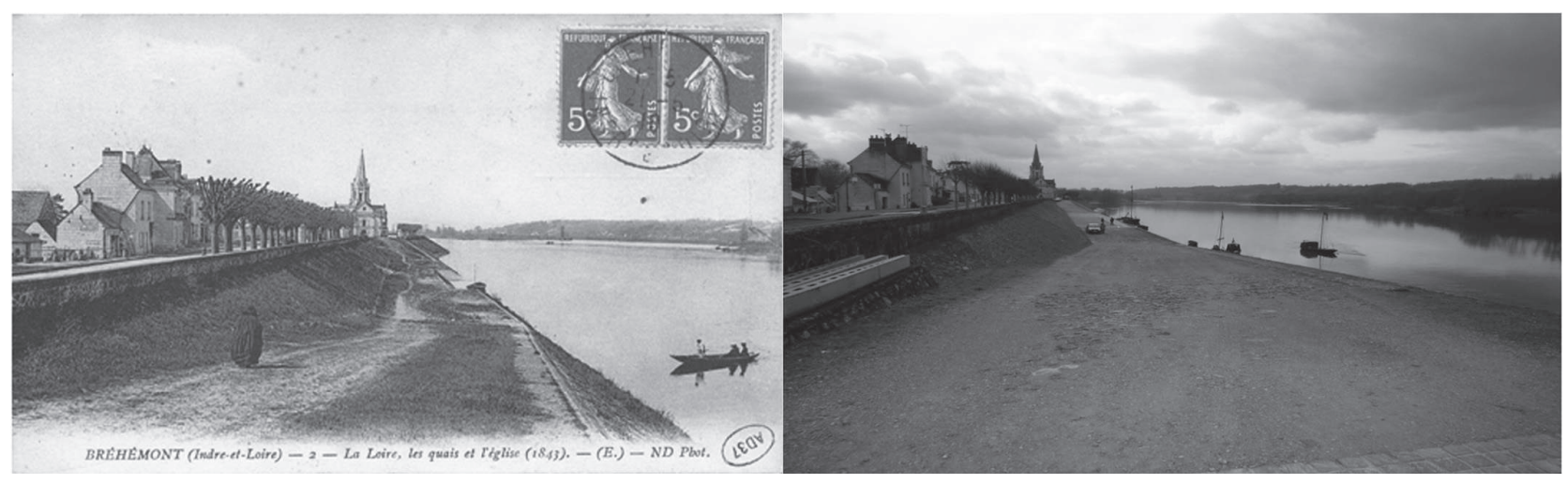

Figure 4 : Port de Bréhémont, fin XIX ${ }^{\mathrm{e}}$ siècle (archives départementales d'Indre et Loire) et actuel Bréhémont harbour, late $19^{\text {th }}$ century (Indre-et-Loire departmental archives)

selon des normes contemporaines et aboutit à une modification du profil de l'ancien port (figure 5).

Bien qu'ayant subit quelques modifications de structure, les ports et quais des deux communes analysées ont donc conservé un aspect général semblable à celui précédent l'arrêt de la navigation. Le panorama de ces ouvrages montre qu'il existe des variantes. D'un côté, la restauration ou la reconstruction des ports s'appuie de manière systématique sur les caractéristiques architecturales et sont assimilés à des programmes de « restauration du patrimoine fluvial ». De l'autre, l'intervention semble parfois conditionnée par l'existence de contextes favorables à l'expression d'une valorisation patrimoniale. Cela ne permet pas dire si les enjeux de valorisation prédominent par rapport à ceux liés à la conservation, ce qui tendrait à confirmer notre hypothèse d'instrumentalisation du patrimoine.
Outre les aspects de forme, la fonction liée à la navigation reste un des objectifs premiers des restaurations quand elles ont lieu. N'occultant pas leur intérêt pour la valorisation du cadre de vie ou les animations culturelles et touristiques, la vision utilitariste des ports et quais reste tenace dans l'esprit des prestataires du tourisme fluvial comme en témoignent les entretiens réalisés. Une des préoccupations les plus communes de ces acteurs est la nécessité de créer un port de carénage et d'entretien. Le site de Rassay-sur-Vienne, situé à proximité du territoire d'étude, fait l'objet d'un projet en ce sens qui a été mentionné à de multiples reprises lors des entretiens. Cette représentation utilitariste du port accompagne la représentation patrimoniale comme le confirme l'analyse des questionnaires réalisés. Les quais et ports sont cités spontanément comme paysage typique du Val de Loire par près de
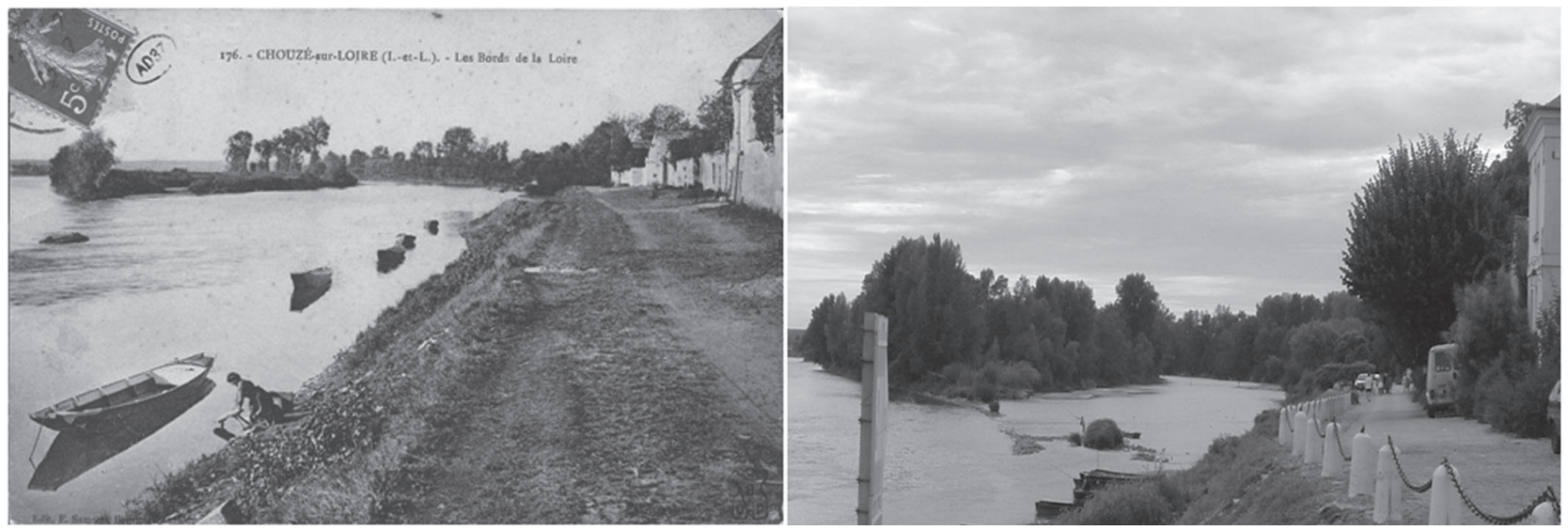

Figure 5 : Port de Chouzé-sur-Loire à la fin du XIx ${ }^{\mathrm{e}}$, début du $\mathrm{XX}^{\mathrm{e}}$ siècle (archives départementales d'Indre et Loire) et aujourd'hui Chouzé-sur-Loire harbour, late $19^{\text {th }}$ century, early $20^{\text {th }}$ century (Indre-et-Loire departmental archives) and nowadays 
$17 \%$ des personnes questionnées; ce chiffre monte à près de $35 \%$ si on agrège à ce résultat le fait de citer des villages structurés et connus par leur port.

Entre cette vision utilitariste et patrimoniale et en considérant que la restauration des ports et quais vise généralement le développement d'usages touristiques, on déduit que les préoccupations associées à ces objets sont liées autant à la conservation qu'à la valorisation. Qu'en est-il des embarcations qui matérialisent la pratique de la navigation?

\section{DiVERSITÉ DES BATEAUX " PATRIMONIAUX » LIGÉRIENS ET PROJETS DE DÉVELOPPEMENT LOCAUX}

Les bateaux « traditionnels » de Loire sont intéressants à étudier du point de vue du processus de patrimonialisation pour plusieurs raisons. Tout d'abord parce que le phénomène dit de "renouveau de la marine de Loire » est antérieur à l'inscription au patrimoine mondial. Amorcé au début des années 1990, il s'étend à l'ensemble du périmètre inscrit au cours de cette même décennie. Par ailleurs, ce phénomène parallèle à l'émergence du mouvement «SOS Loire vivante » est considérée par plusieurs auteurs (Huyghues Despointes, 2008, Verdelli, 2008, Pin, 2010) comme une des composantes ayant conduit à faire émerger la dimension patrimoniale du Val de Loire. Une autre raison est l'utilisation de la batellerie au cours du processus de patrimonialisation, et spécifiquement lors de la dernière étape qui est la valorisation (Di-Méo, 2008). Il est donc pertinent d'observer les bateaux, dans leur diversité et leurs contextes locaux, afin de mieux comprendre leur rôle dans les nouveaux projets de développement.

Concernant les expériences de reconstruction de bateaux ligériens s’inspirant de l'architecture navale fluviale ligérienne, il apparaît qu'elles sont liées à des travaux d'érudits et de passionnés. Parmi les personnes s'étant consacrée à cette tâche, François Beaudouin, ancien conservateur du musée de la batellerie de Conflans-Sainte-Honorine, a écrit de nombreux ouvrages et articles consacrés aux bateaux de Loire (Beaudouin, 1984, 1985, 2003). C'est en s'appuyant sur ces travaux que François Beaudouin a dirigé la reconstruction récente d'un scute de Loire, forme de bateau médiéval, pour laquelle il a ainsi défini les outils, les types de bois, les techniques de construction ${ }^{3}$. Cette embarcation a complété la flotte d'une association locale de la vallée du Cher (Savonnières) qui participe de la construction d'un paysage fluvial singulier. Toutefois cette expérience, où l'effort de recherche réalisé est important, s'avère tout à fait singulière en Val de Loire. Les bateaux sont le plus souvent reconstruits selon quelques éléments identitaires.

Ainsi en est-il par exemple à Bréhémont et Chouzé-sur-Loire où la situation est bien représentative des expériences de reconstruction menées en Loire moyenne. On y recense des bateaux dont l'architecture s'apparente de manière assez proche à celle des anciens bateaux de Loire $^{4}$. Il s'agit principalement de bateaux d'associations de marine de Loire, comme «la matelote de Bréhémont » (figure 6a) et pouvant être financés en partie par la collectivité publique, comme à Chouzé-sur-Loire ${ }^{5}$. Dans ce cas précis, il existe un lien avec l'animation culturelle puisque le musée de la batellerie de cette commune organise des sorties thématiques à bord du bateau. La batellerie devient ici à la fois moyen de découverte tout autant que thématique de médiation patrimoniale. Outre cette expérience, le site de Chouzé-sur-Loire démontre également comment les bateaux peuvent être utilisés de manière indirecte dans la construction de l'image de la commune. Ainsi, la plate de Loire, barque traditionnelle, est intégrée au mobilier urbain notamment sous la forme d'un parterre fleuri en entrée de bourg (figure 6b).

Notons qu'il existe par ailleurs des projets de reconstruction de bateaux à passagers à l'architecture moderne ${ }^{6}$ mais habillés d'un bardage bois rappelant l'esthétique des bateaux traditionnels. C'est le cas à Candes-Saint-Martin, commune localisée à l'extrémité aval du terrain d'étude, où la recherche d'une certaine authenticité n'est pas l'objet central de la construction, elle n'a d'ailleurs absolument pas été revendiquée en tant que telle, du moins lors de l'entretien que nous avons pu mener. Néanmoins,

\footnotetext{
3. «Toute ma vie j'ai travaillé sur les bateaux traditionnels et plutôt anciens.. mais sur le scute lui-même, j'ai travaillé plusieurs années dessus... récolté des centaines d'épaves de bateaux divers afin d'éclairer l'architecture de ce dernier. »(Entretien avec François Beaudouin, 2010.)

4. Présence d'un fond plat, d'une proue à levée, construction à clin, etc.

5. Cofinancements incluant un Leader + du pays chinonais et une subvention de la municipalité.

6. Coque aluminium pour l'un, coque en fibre pour l'autre avec des profils inspirés de bateaux maritimes.
} 


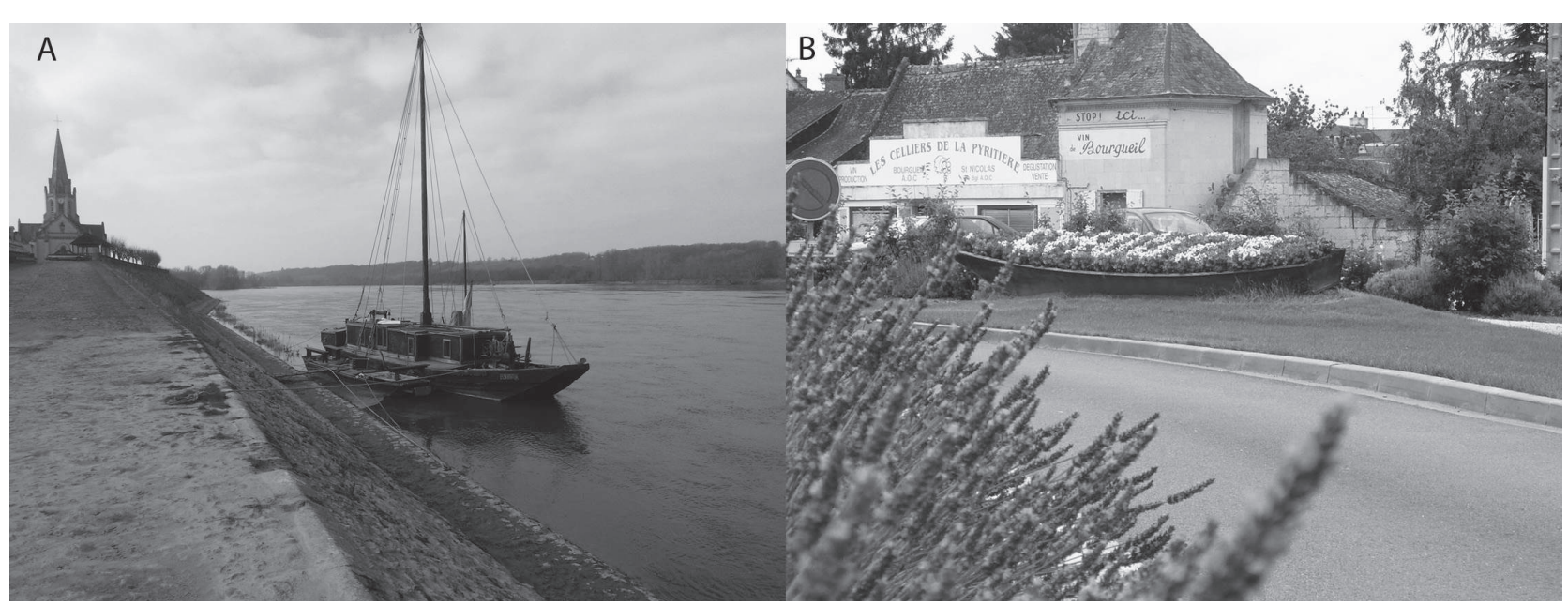

Figure 6 : Bateau traditionnel à Bréhémont (a) et Plate de Loire utilisée en tant que mobilier urbain à Chouzé-sur-Loire (b) (Crédit photographique : Benoit Pin)

Traditional boat in Bréhémont (A) and "plates de Loire" used as street furniture in Chouzé-sur-Loire (B) (Photo credit: Benoit Pin)

l'habillage de ces bateaux traduit le rôle de ressource que peut être aujourd'hui celui de la batellerie traditionnelle. Il s'agit ici de profiter de l'image de cette marine de Loire tout en s'affranchissant de ses contraintes.

Le panorama des bateaux traditionnels ligériens présenté ici démontre une grande variété alors qu’il est fait référence à la batellerie du XVIII ${ }^{\mathrm{e}}$ siècle. La grande étendue de ce nuancier apparait logique eu égard à la mutation d'usage de ces bateaux qui a conduit leur patrimonialisation. Toutefois, et même si les formes sont variées, l'analyse des questionnaires auprès des habitants, a permis de mettre en évidence l'absence de distinction entre les différentes catégories de bateaux. En revanche, les bateaux identifiés comme étant traditionnels semblent constituer, pour ces habitants, un des motifs paysagers typiques du Val de Loire. Ce constat renforce le caractère de ressource de ces bateaux en mettant l'accent sur leur apport au paysage local qui est de nature équivalente à celle des châteaux pour les personnes questionnées. Ces deux images, celles du château et du bateau traditionnel (type chaland, toue, futreau), figurent d'ailleurs parmi les plus utilisées au sein des documents de communication des professionnels et institutions du tourisme (Pin, 2010).

Ces résultats montrent l'étendue des formes de reconstruction et une variété de considération de la dimension patrimoniale qui est mobilisée pour des actions de développement local. De même, la participation des bateaux à la (re)construction d'un paysage fluvial apparait en toile de fond des différents exemples abordés. Son apport est plus particulièrement revendiqué pour le site de Bréhémont que nous allons détailler plus spécifiquement.

\section{Action publique et VAlorisa- TION DE LA BATELLERIE (PORTS ET BATEAUX), VERS UN MODÈLE DE DÉVELOPPEMENT LOCAL ORIGINAL}

De l'analyse des exemples précédents, il ressort comme tendances que le patrimoine associé aux aménagements portuaires fait l'objet de restaurations ponctuelles. Elles transigent peu avec les caractéristiques des anciens ouvrages ce qui n'est pas le cas de la batellerie où les chantiers de reconstructions ne suivent pas tous la même logique. Par contre ils offrent de réelles potentialités touristiques intégrées aux projets de développement locaux. Afin de conforter ces observations, nous allons nous intéresser aux valorisations réalisées à l'échelle communale et départementale. Il faut souligner que l'action développée au sein du conseil général est spécifique à l'Indre-et-Loire (traditionnellement ce sont des actions du Comité Départemental de Tourisme) ce qui démontre une réelle ambition de valorisation du patrimoine fluvial.

À Bréhémont, la politique de développement urbain a connu une forte évolution depuis la nouvelle politique de prévention du risque d'inon- 
dation qui privilégie la maîtrise de l'urbanisation en zone inondable (Servain et Yengué, 2012). L'ancien modèle de développement assis sur une croissance de logements a été remplacé par une politique construite autour de la valorisation du caractère inondable de la commune, renversant les contraintes de la commune pour en faire des atouts.

Aussi, le projet de développement communal a été fondé sur la valorisation du patrimoine et en particulier celui de la batellerie. Cela s'est traduit par une restauration du port, financée en partie par le Département, mixant le respect de l'architecture ancienne et répondant aux attentes nouvelles relatives aux activités nautiques (navigation de l'association locale, proposition de sorties sur bateau traditionnel, pédalo). Était projetée la création, par la communauté de communes d'Azay-le-Rideau, d'un centre d'interprétation présentant la culture du chanvre, liée au caractère inondable, et la batellerie ce qui démontrait une réelle volonté de valorisation d'un patrimoine local singulier constituant une ressource territoriale spécifique. Le conseil général a également prévu la création d'une "Maison de la Loire » devant sensibiliser sur le «bien vivre » en zone inondable. Comme ces projets l'illustre, la batellerie est rarement valorisée seule et participe d'un ensemble plus complet constituant une offre locale originale. À l'échelle départementale, le patrimoine lié aux aménagements portuaires et à la batellerie est suffisamment dense sur le linéaire ligérien pour faire l'objet d'une action spécifique. Celle-ci est centrée sur la valorisation plus que la conservation bien que ce critère soit pris en compte pour le choix des projets financés.

Par ailleurs, cet exemple permet de confirmer que le patrimoine portuaire et batelier est souvent soutenu pour sa fonction de mise en scène fluviale en lien avec le paysage. L'analyse des documents de communication de deux actions atteste également de cette orientation : l'événementiel festif ligérien, "Jour de Loire », et le label touristique «Touraine Loire Valley », développé par le Comité Départemental de Tourisme d'Indre-et-Loire. L'analyse de la programmation de « Jour de Loire », créée en 2002 par le conseil général du Maine-etLoire et repris ensuite par le département d'Indreet-Loire, montre que la batellerie est un élément moteur, notamment pour les communes situées dans le secteur d'étude ${ }^{7}$. Ceci passe notamment par l'utilisation des bateaux sous forme de scène ou de décors. L'objet y est détourné de sa vocation première et devient une ressource à l'image forte. Cette manifestation donne un bon aperçu de la manière dont le patrimoine est valorisé et constitue une des expressions du discours de réconciliation avec le fleuve, largement répandu à l'échelle du bassin, et transparaissant dans les actions du conseil général d'Indre-et-Loire.

Dans le cas de l'évènement «Touraine Loire Valley ", les documents de communication confirment l'idée d'un patrimoine de Loire à valoriser plus qu'à préserver. Le style narratif adopté en est une manifestation très claire de la volonté de construire et d'utiliser l'image patrimoniale (Di Méo, 2008). Cela est de nouveau le cas dans l'exemple suivant, tiré de la plaquette Touraine Loire Valley - Ici, vivre est un art : "La surprise? C'était un tour en bateau. En futreau même! Quand on s'est réveillé le lendemain du pique-nique, Maman nous a annoncé la grande nouvelle. On allait faire une balade sur la Loire, à bord d'un bateau en bois typique de la région. » Or, la diversité des bateaux traditionnels démontre que leur typicité peut être largement discutable. Ces documents illustrent à eux seuls à quel point ces bateaux, dans leur grande diversité, ont finit par intégrer les motifs paysagers du Val de Loire au sens d'Augustin Berque (Berque, 1995). Il s'agit là d'une traduction de leur rôle actuel dans la valorisation des territoires locaux. Enfin, à travers une vision largement idéalisée, ils suggèrent l'idée d'une symbiose entre l'Homme et le fleuve; un art de vivre ligérien.

La batellerie est donc un exemple pertinent de la manière dont le patrimoine peut être mobilisé. Elle constitue un très bon support de mise en tourisme et d'animation culturelle. Sa réapparition au cours de la dernière étape du processus de patrimonialisation qu'est la valorisation démontre le côté construit du processus, l'instrumentalisation du patrimoine. Enfin, l'observation des différents contextes a permis de mettre en évidence que les bateaux, mais également le paysage portuaire, participent de ressources territoriales plus complexes à l'échelle locale. En dépit de la diversité des offres touristiques des différents territoires, ces deux

7. À Bréhémont, les fêtes organisées mobilisent systématiquement l'association locale et ses bateaux. 
formes de patrimoine demeurent une constante en Val de Loire ce qui tend à démontrer l'existence d'un modèle de développement local alternatif commun à l'échelle de ces territoires ruraux de vallée.

\section{Conclusion}

L'objet de cet article était d'interroger l'intérêt porté à la dimension patrimoniale des aménagements portuaires et bateaux traditionnels de Loire, lesquels participent à l'offre touristique originale des territoires prospectés. Il s'agissait ici de souligner les objectifs poursuivis et par extension de confirmer la prégnance d'un modèle de développement local alternatif, mobilisant une ressource territoriale construite.

De l'analyse du contexte historique et des restaurations et de reconstruction de ports et de bateaux, il ressort deux tendances générales. D’un côté, le patrimoine associé aux aménagements portuaires fait l'objet de restaurations respectant les caractéristiques de l'ancien, mariant la fonction utilitariste initiale à des fonctions de cadre de vie, de développement de nouvelles pratiques, de mise en paysage et de support d'événementiels touristiques et culturels. De l'autre, la batellerie fait l'objet de reconstructions variées conçues pour de nouveaux usages.

Le point commun de ces deux types de patrimoine est leur intégration constante aux projets de développements locaux émergeants sur le linéaire du terrain d'étude et même au-delà. Leur participation systématique à l'offre touristique et culturelle locale souligne leurs fortes potentialités. Ceci est renforcé par des actions supra-locales telles que celles du département d'Indre-et-Loire qui participe à l'élan local au travers de diverses aides.

De l'observation des différents contextes il ressort que l'intérêt porté à ces types de patrimoine réside d'abord dans la valorisation plus que dans l'enjeu de conservation. Il s'agit là d'un constat confirmant d'autres modèles de développement constatés par ailleurs, mais qui prend un sens singulier dans le cadre d'un site inscrit au patrimoine mondial, le Val de Loire.

\section{Remerciements}

Les travaux ayant donné lieu à cet article ont été menés à l'UMR 7324 CITERES (CNRS- Université de Tours) et sont soutenus par la Zone Atelier Loire et pour certains financés par la plateforme recherche du Plan Loire (FEDER-Etablissement Public Loire), dont le projet PATRA (patrimoine et trajectoires paysagères).

\section{Bibliographie}

Beaudouin F., 1984. Batellerie et bateaux du bassin de la Loire: La marine de Loire et son chaland, Les cahiers du musée de la Batellerie, $\mathrm{n}^{\circ} 12$, Conflans-Sainte-Honorine, $40 \mathrm{p}$.

Beaudouin F., 1985. Chaland de Basse-Loire, gabarot de mayenne, bateau nantais, Les cahiers du musée de la Batellerie, $n^{\circ} 12$, Conflans-Sainte-Honorine, 36 p.

Beaudouin F., 2003. L'archéologie nautique ligérienne, 303, arts, recherches et créations, $\mathrm{n}^{\circ} 75$, p. 57-65.

Berque A., 1995. Les raisons du paysage: de la Chine antique aux environnements de synthèse, Paris, Éditions Hazan, $192 \mathrm{p}$.

Bonnefond M., Servain-Courant S., Verdelli L., 2009. Politiques paysagères en action pour la protection et la valorisation du patrimoine naturel et culturel; application au cas de Candes-Saint-Martin et de la Brenne (Région CentreFrance), Projets de paysage, Dossier thématique $\mathrm{n}^{\circ} 2$, le 26 juin 2009, 11 p.

Brunel S., 2007. Tourisme et mondialisation, Vers une disneylandisation universelle? Géographie, ${ }^{\circ}$ 1525, juin 2007, p. 12-30.

Cayla P., 2007. Fréquenter le patrimoine culturel sociétal ligérien, l'expérience de l'Ecomusée de Montjean Loire Angevine - référence pour des enjeux patrimoniaux à l'échelle du bassin fluvial, $14 \mathrm{p}$.

Chevalier B., 1986. Les villes de la Loire, dans Vigier Ph., Une histoire de la Loire, Ramsay, p. 170-194.

Chiappero D., 1996. Patrimoine ligérien et développement urbain: définition et nouvelles pratiques d'aménagement, Doctorat, Université François-Rabelais de Tours, Centre d'Études Supérieures en Aménagement, 514 p.

Chony F., 1992. L'Allégorie du patrimoine, Paris, Seuil, 272 p.

Davodeau H., 2004. La patrimonialisation : un vecteur d'appropriation des vallées ligériennes?, Norois, n 192, p. 63-69.

Di Méo G., 2008. Processus de patrimonialisation et construction des territoires, dans Patrimoine et industrie en PoitouCharentes : connaître pour valoriser, Poitiers - Châtellerault, $19 \mathrm{p}$.

Dion R., 1961. Histoire des levées de la Loire, Imprimerie Habauzit, 312 p.

Durrenberger V., 1999. Les aménagements en lit mineur : ports, épis, moulins, pêcheries entre Tours et Saumur au Moyen Âge, Mémoire de maîtrise, université de Tours. 
Greffe X., 1999. La gestion du patrimoine culturel, Anthropos, $262 \mathrm{p}$.

Fouillet N., David S., Delemont M., Gardere P., Yvernault F., 2011 . Rapport final d'opération de fouille archéologique préventive, La fouille de la Clinique des Dames Blanches, Orléans, Service régional de l'Archéologie de la région Centre.

Gumuchian H., Pecqueur B., 2007. La ressource territoriale, Paris, Économica, 252 p.

Huyghues-Despointes F., 2008. Des barrages au patrimoine mondial : la Loire comme objet, d'action publique, Thèse de Doctorat, université François-Rabelais, 443 p.

Jeanson D, Mantellier P., 1987. Histoire de la communauté des marchands fréquentant la rivière de Loire et fleuves descendant en icelles, Denis Jeanson, Tours, 696 p.

Kaufmann J.-C., 2001. L'entretien compréhensif, Nathan, Paris, $128 \mathrm{p}$.

Landel P.-A., Senil N., 2009. Patrimoine et territoire, les nouvelles ressources du développement, Développement durable et territoires, Dossier 12 : identités, patrimoines collectifs et développement soutenable, $15 \mathrm{p}$.

Le Nevez N., Mauret-Cribellier V., Clericy O., 2007. Recensement des cales et des quais sur la Loire et ses affluents anciennement navigués, Archéologies en Loire. Actualité de la recherche dans les régions Centre et Pays-dela-Loire, Aestuaria, Cordemais, p. 89-96.
Nora P., 1992. L'ère de la commémoration, dans Nora Pierre, Les lieux de mémoire, Gallimard, Paris, p. 4687-4719.

Pecqueur B., 2006. Le tournant territorial de l'économie globale, Espaces et sociétés, nº 124-125, p. 17-32.

PIN B., 2010. De la conception d'un fleuve patrimonial à sa mise en tourisme : du Val de Loire au flewve Niger, Thèse de Doctorat, université François-Rabelais, 446 p.

République française, 2000. Proposition d'inscription Val de Loire au patrimoine mondial de l'UNESCO paysages culturels, $553 \mathrm{p}$.

Servain-Courant S., Yengué J.-L., 2012. Les forêts du Val de Loire: Maillon dans la construction d'un paysage emblématique. Le cas de Bréhémont en Indre et Loire, dans Convol A., Forêt et Paysage, Paris, Harmattan, p. 131-140.

Verdelli, L., 2008. Héritages fluviaux, des patrimoines en devenir - Processus d'identification, protection et valorisation des paysages culturels en France, Portugal et Italie: quelques exemples significatifs, Thèse de Doctorat, universités de Tours et de Coimbra, 680 p.

Verdier M., Guilbaud R.-C., 2003. Proposition d'aménagements ponctuels de ports de Touraine. Rapport de synthèse, Tours, Direction de la Culture et des Sports, Service du Patrimoine Culturel, conseil-général d'Indre-et-Loire, fascicules, feuillets, $7 \mathrm{p}$. 